\title{
263 The soft palate
}

A The palatine aponeurosis is formed by the expanded tendons of levator palati muscles.

B Tensor palati turns through 90 degrees around the pterygoid hamulus.

C Contains mucous glands, lymphoid tissue and taste buds.

D Is lined by squamous epithelium on its superior surface.

E Main blood supply comes from the greater palatine artery.

\section{Derivatives of the pharyngeal pouches}

A The intratonsillar cleft is a remnant of the second pouch.

B Parathyroid tissue develops from the fourth and fifth.

C The ultimobranchial body is a fifth pouch derivative.

D Branchial cysts usually lie posterior to sternomastoid.

$E$ The thyroid gland is derived from the fourth pouch.

\section{White lesions of the oral cavity}

A Leucoplakia can be rubbed off.

B Candidiasis may appear as a red or white area.

C Vitamin B12 deficiency causes white patches on the tongue.

D A pattern of red areas with white or yellow elevated borders which changes from day to day should be biopsied.

E Lace-like pattern is characteristic of lichen planus. 